Diagnostic: surcharge administrative en constante progression. Nos patients aimeraient davantage d'attention et de temps de notre part à nous, médecins, alors que la régulation croissante nous impose toujours plus de documentation et d'administration. Des mesures doivent être prises de toute urgence avant que nous soyons réduits à traiter des radios et des résultats de laboratoire et que le niveau de frustration explose. Dans cet article, le DrVolker Schade présente un instrument visant à réduire la charge administrative des médecins et à mieux répartir les tâches. Mais les analyses individuelles et les conseils personnalisés restent ici aussi primordiaux.

\title{
Retour au cœur de l'activité médicale
}

\author{
Volker Schade \\ Dr, Centrum für PersonalManagement und Organisationsgestaltung Bern (cpmo)
}

La charge administrative des médecins hospitaliers (ci-après: médecins) est élevée. Le temps qui y est consacré est du temps perdu pour la prise en charge directe du patient. La dernière étude concomitante sur les répercussions du nouveau financement hospitalier, mandatée par la FMH, a révélé que la charge administrative des médecins continue d'augmenter. Le BMS a publié plusieurs articles à ce sujet. Il ne fait aucun doute qu'il s'agit là d'un thème brûlant d'actualité. En outre, la tendance à une augmentation des tâches administratives des médecins semble s'être stabilisée ces dernières années et aucun inversement de cette tendance n'est en vue. Des idées créatives s'imposent donc pour gérer cette problématique. Une approche maintes fois éprouvée est présentée ci-dessous.

\section{Une administration accrue qui n'en finit pas?}

L'énorme investissement des médecins hospitaliers est une réalité. Malgré des dispositions légales, les médecins continuent de faire un nombre trop important d'heures supplémentaires. L'enquête menée en 2014 par l'ASMAC auprès de ses membres sur la charge de travail des médecins-assistants et des chefs de clinique a révélé, en effet, que la durée de travail hebdomadaire moyenne dépassait de 6,5 heures la limite hebdomadaire maximale de 50 heures [1]. Un médecin-assistant ou un chef de clinique sur quatre a même travaillé en moyenne plus de 60 heures par semaine. La durée de travail hebdomadaire fixée dans le contrat n'est respectée que pour $29 \%$ des médecins [2]. Ce sont là des signes alarmants du point de vue des dispositions légales et de la charge de travail. L'ASMAC recommande entre autres de décharger les médecins des tâches administratives [3].

Des études montrent que les tâches administratives représentent une part (trop) importante du travail des médecins. Létude concomitante sur SwissDRG citée plus haut a analysé l'investissement en temps des médecins en soins somatiques aigus. La part des activités centrées sur le patient ne représente plus qu'un tiers du temps de travail d'un médecin [4]. Et cette part a fortement diminué depuis 2011. Malheureusement, la charge administrative a connu pendant cette même période une évolution inverse. Du temps perdu donc pour les activités médicales importantes. Même la NZZ a récemment traité ce sujet en titrant «Mediziner versinken in der Papierflut» («Les médecins croulent sous la paperasse») [5]. Les médecins-assistants sont particulièrement concernés.

Quelles sont les conséquences de cette lourde charge administrative? 


\section{Conséquences de la surcharge de travail}

Cette lourde charge affecte aussi bien la santé physique que psychique des médecins. $11 \%$ des médecins hospitaliers ayant participé à la dernière étude concomitante sur SwissDRG ont estimé que leur santé physique était tout juste suffisante. $3 \%$ des médecins ont indiqué souffrir souvent ou la plupart du temps d'une dépression et environ $80 \%$ des médecins se sentent continuellement sous pression. Or un état de santé physique et psychique insuffisant chez les médecins a des conséquences graves pour les patients, puisque la qualité des soins diminue. Les médecins eux-mêmes le confirment. Seul un cinquième des médecins estiment que la lourde charge de travail ou le manque de temps entravent rarement ou jamais la qualité des soins [6]. La probabilité de complications chez les patients augmente. $38 \%$ des médecins interrogés déclarent avoir mis en danger un patient au moins une fois au cours des deux dernières années suite à un surmenage professionnel [7].

La formation postgraduée des médecins-assistants souffre elle aussi de cette surcharge. En effet, seul un médecin-assistant sur deux est en mesure de suivre sa formation postgraduée de façon satisfaisante pendant son temps de travail [8]. La pénurie de médecins accentue encore plus cette problématique.

\section{Tâches illégitimes}

Quelles répercussions le déséquilibre décrit entre le cœur de métier d'un médecin et les «activités annexes» peut-il avoir? Les tâches administratives notamment sont souvent vécues par les médecins comme «illégitimes». On entend par là des activités considérées comme déraisonnables voire inutiles pour l'exercice de sa propre fonction. Les tâches illégitimes ne respectent pas l'identité professionnelle, mais exigent au contraire d'accomplir des activités qui ne correspondent pas à l'essence même du rôle professionnel. Un facteur de stress qui conduit à une mise en danger de l'estime de soi.

Des rapports ont pu être établis entre les tâches illégitimes et les indicateurs de santé psychique. Une grande quantité de tâches illégitimes engendre des sentiments négatifs vis-à-vis de l'organisation, des troubles psychosomatiques ainsi qu'une diminution de la satisfaction au travail [9].

Cela paraît évident: la part élevée de tâches administratives des médecins, qui ne cesse d'augmenter, représente une problématique sérieuse qui engendre toute une série de problèmes. La conséquence logique de ce phénomène est également une augmentation des coûts pour les hôpitaux. Il est donc urgent de dévelop- per des solutions permettant de réduire la charge administrative des médecins, lesquelles devraient aussi avoir des conséquences favorables sur les coûts.

\section{Une approche: la gestion des hospitalisations}

L'administration dans les hôpitaux ne doit pas être diabolisée. Elle procure en effet de l'ordre et une sécurité à l'organisation. Mais elle peut aussi porter atteinte aux intérêts de l'entreprise et des personnes qui y sont employées. C'est le cas par exemple lorsque des médecins doivent accomplir toujours plus de tâches qui n'ont rien à voir avec l'activité médicale. Ce n'est pas pour ces tâches que la grande majorité des médecins ont choisi leur profession.

En substance, les tâches "non médicales» devraient donc être confiées, dans la mesure du possible, à des collaborateurs spécialement formés.

Notre intérêt accru pour ce thème a été motivé par des efforts déployés en vue de réduire la durée de travail des médecins, qui n'était plus conciliable avec les réglementations sur le temps de travail. L'Hôpital de l'Ile de Berne a joué ici un rôle de précurseur au début des années 2000. Un concept y a été développé en collaboration avec des spécialistes internes et externes pour soulager les médecins de manière ciblée. Les médecins ont confié les activités administratives chronophages à un collaborateur administratif. Ce procédé a eu tellement de succès que nous avons continué à le développer.

La "gestion des hospitalisations» était née. La principale tâche du / de la gestionnaire des hospitalisations consiste à s'occuper du dossier du patient et de l'organisation liée aux activités nécessaires pour le patient avant, pendant et après l'hospitalisation (voir tableau 1). Ainsi soulagés, les médecins bénéficient d'un gain de temps. En outre, le gestionnaire des hospitalisations sert d'interlocuteur aux services internes et externes.

Tableau 1: Principales tâches du gestionnaire des hospitalisations.

Préparation de l'admission, p. ex.

- constituer le dossier d'admission du patient

- préparer l'admission à l'hôpital

Tâches (administratives) concernant le patient pendant son hospitalisation, p. ex.

- compléter le dossier du patient

- réclamer rapports et résultats

- organiser les examens médicaux et les entretiens avec les spécialistes

Préparation de la sortie, p. ex.

- coordonner la gestion administrative de la sortie avec les services concernés

- organiser la réadaptation 


\section{Implémentation de la gestion des hospitalisations}

Dans le cadre de notre activité scientifique dans les hôpitaux suisses, nous avons été de plus en plus confrontés ces quinze dernières années au problème de la charge administrative des médecins et à la manière de la réduire. Lors de la restructuration de la répartition des tâches, la gestion des hospitalisations a été introduite avec succès dans différents hôpitaux et cliniques. La figure 1 montre le déroulement d'un projet de gestion des hospitalisations.

Dans un premier temps, la situation spécifique de la clinique ou de l'hôpital est examinée à l'aide d'un questionnaire, d'interviews ou d'observations. Sur cette base, des objectifs sont élaborés pour chaque clinique. Des possibilités d'allègement concrètes pour les médecins sont proposées et le catalogue de tâches du gestionnaire des hospitalisations est dressé. Un gestionnaire des hospitalisations est ensuite recruté et initié à sa mission. Tel est le déroulement en quelques mots. Quel en est le gain?

\section{Gestion des hospitalisations: réduire la charge administrative des médecins!}

L'évaluation des projets mis en place jusqu'ici révèle des résultats prometteurs. Ainsi, une clinique est parvenue à réduire le temps de travail de 35 minutes par jour et par médecin-assistant malgré l'augmentation parallèle du volume de travail [10]. La gestion des hos-

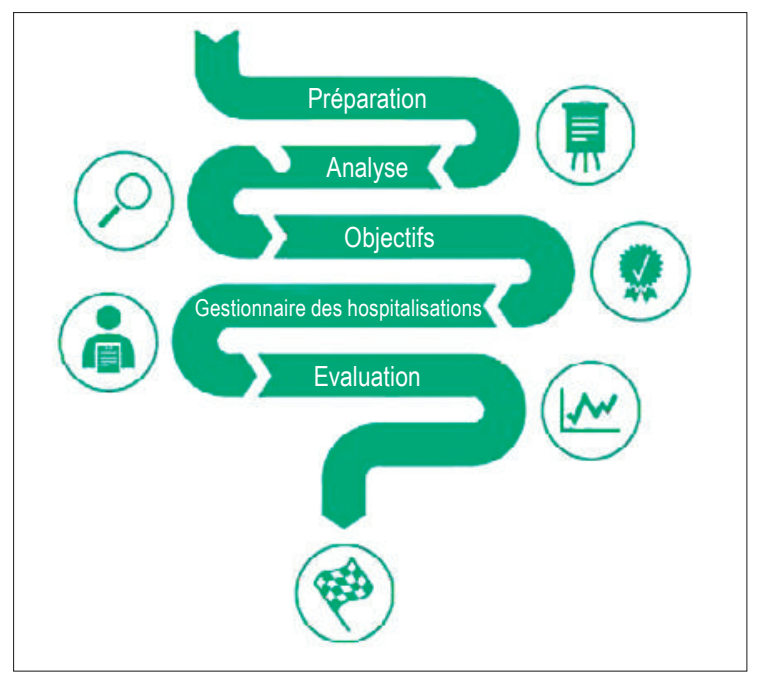

Figure 1: Déroulement de l'implémentation de la gestion des hospitalisations.

pitalisations a permis de réduire, parfois même de diviser par deux, la part des tâches administratives des médecins-assistants dans tous les projets évalués. Cela a généré un important gain de temps pour les principales activités médicales comme le travail avec le patient, la formation postgraduée ou la recherche. Des compétences mieux délimitées, un travail plus efficace et un moindre morcellement de l'activité médicale font partie des autres améliorations obtenues par la gestion des hospitalisations (voir figure 2). La plus grande disponibilité et la meilleure actualisation des dossiers médicaux sont un autre aspect particulièrement apprécié par les médecins.

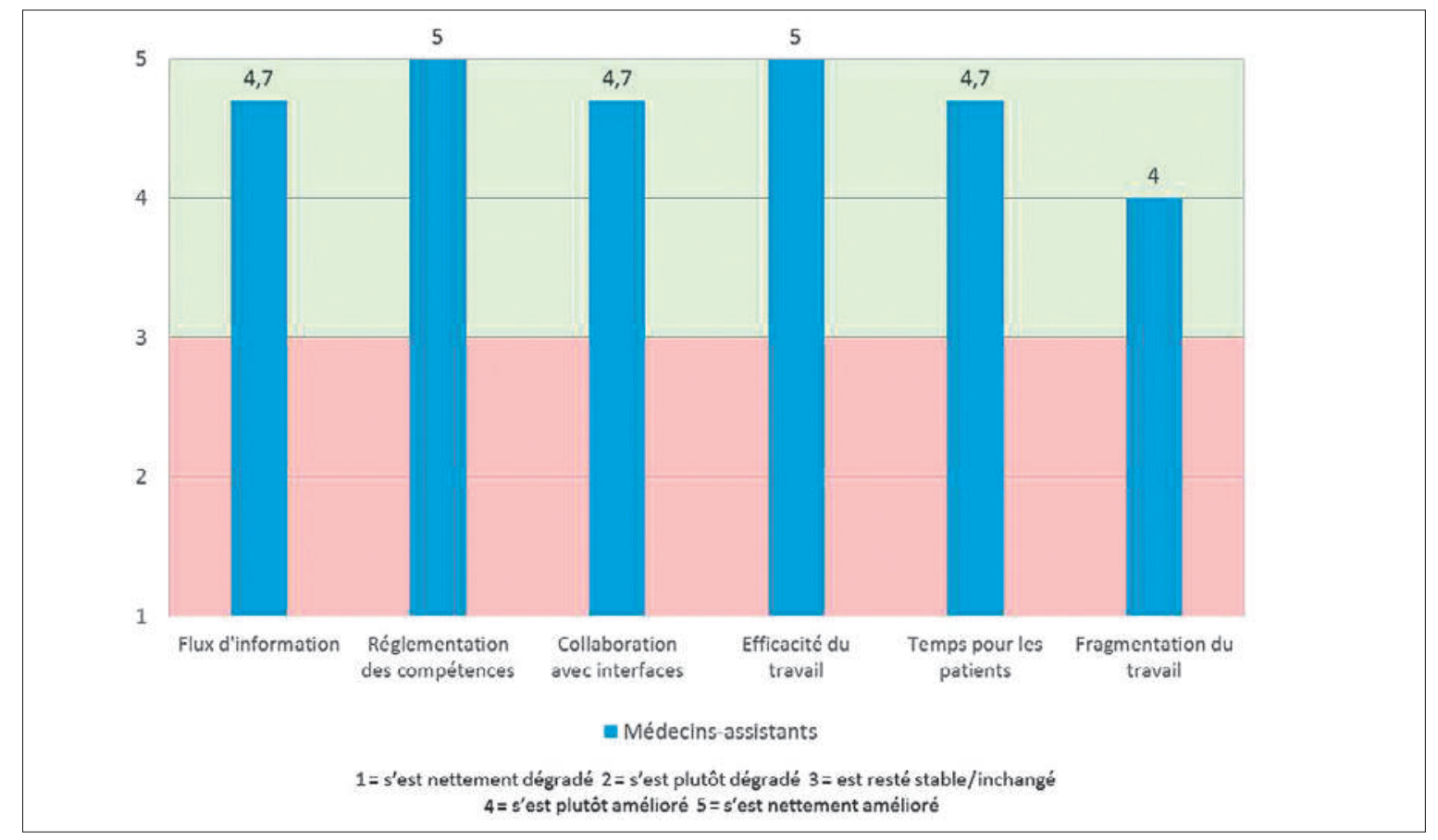

Figure 2: Estimation des changements occasionnés par la gestion des hospitalisations (graphique: extrait, modifié d'après Schade $\mathrm{V}$ et al. [10]). 
D'autres groupes professionnels profitent également de la gestion des hospitalisations. Les services infirmiers et ambulatoires ont accompli moins de tâches administratives pour les médecins (p. ex. formulaires, organisation d'examens et d'entretiens avec les spécialistes, photocopies, etc.). La gestion des hospitalisations a contribué à améliorer la collaboration au sein des cliniques et avec les intervenants externes.

Plusieurs évaluations de projets ont montré que la situation de travail s'était largement améliorée dans les cliniques ou hôpitaux en question suite à l'introduction de la gestion des hospitalisations. Hormis les améliorations souhaitées, ces évaluations ont également révélé une augmentation de la satisfaction au travail des médecins. C'est là un aspect non négligeable au vu des énormes défis que représente l'activité médicale. Par ailleurs, la gestion des hospitalisations permet de créer des emplois attrayants. Des postes qui sont beaucoup plus faciles à pourvoir que des fonctions médicales.

On peut donc dire, pour simplifier, que la répartition des tâches liées au patient dans les cliniques est en train de rentrer dans l'ordre.

\section{Conclusion}

Une entière satisfaction donc? Pour les médecins et autres groupes professionnels concernés, c'est effectivement le cas, d'après les témoignages recueillis. Néanmoins, la gestion des hospitalisations coûte de l'argent. Des coûts qui viennent visiblement grever les budgets. Toutefois, les améliorations décrites plus haut laissent penser qu'elles permettent d'économiser encore plus de coûts.

Les efforts déployés pour alléger la charge administrative des médecins et mieux répartir les tâches présentent différentes facettes. Dans certaines institutions, on observe un élargissement des tâches des secrétariats des services ou la transmission de tâches au personnel infirmier. Or, confier à ces groupes professionnels déjà surchargés des missions supplémentaires n'est pas sans problèmes. Par ailleurs, ces transferts de tâches sont rarement accompagnés de la base conceptuelle nécessaire.
La formation des secrétariats est suffisante pour la partie administrative des tâches relevant de la gestion des hospitalisations, celle du personnel infirmier pour la partie médicale. Mais c'est d'une combinaison des deux que nous avons besoin. Cela implique donc une nouvelle fonction clairement définie. La gestion des hospitalisations aborde les problèmes existants en les intégrant dans un concept éprouvé.

«Le gestionnaire des hospitalisations nous libère du temps que nous pouvons consacrer à d'autres choses comme l'examen des patients et des entretiens avec les proches» (déclaration d'un médecin).

Tout est dit.

\section{Références}

1 ASMAC. Charge de travail des médecins-assistant(e)s et chef(fe)s de clinique. Enquête des membres, 2014. (en allemand seulement) www2.vsao.ch/fileupload/201449144848 pdf.pdf

2 ASMAC. Charge de travail des médecins-assistant(e)s et chef(fe)s de clinique. Management Summary, 2014. www2.vsao.ch/ fileupload/20144148397 pdf.pdf

3 ASMAC. Conditions de travail illégales pour les médecinsassistants et chefs de clinique. Communiqué de presse, 2014 www2.vsao.ch/fileupload/2014710141919_pdf.pdf

4 Meyer B, Rohner B, Golder L, Longchamp C. Hausse continue des tâches administratives: Bulletin des médecins suisses. 2016;97(1): 6-8.

5 Hehli S. Zu viel Bürokratie : Mediziner versinken in Papierflut. NZZ, 2016. www.nzz.ch/schweiz/mediziner-versinken-in-derpapierflut-1.18672428

6 Golder L, Longchamp C, Jans C, Tschöpe S, Venetz A, Hagemann M, Schwab J. Motivation constante du corps médical hospitalier malgré une bureaucratisation croissante: Rapport final de l'étude concomitante de gfs.bern (version longue, seulement en allemand), 2015. www.fmh.ch/files/pdf17/20160106_FMH-Begleit forschung_Schlussbericht_D.pdf

7 Schoch C. Assistenz- und Oberärzte arbeiten zu lange: Gefahren für die Sicherheit der Patienten. NZZ, 2014. www.nzz.ch/schweiz/ gefahren-fuer-die-sicherheit-der-patienten-1.18283852

8 Siegrist M, Gutscher H, Giger M. Was haben Betriebskultur, Motivation und Fremdjahr mit der Weiterbildung zu tun? Die wichtigsten Ergebnisse der Assistentenumfrage 2003: Bulletin des médecins suisses 2004; 85 (15): 783-91.

9 Semmer NK, Jacobshagen N, Meier LL, Elfering A, Kälin W et al. Psychische Beanspruchung durch illegitime Aufgaben. Immer schneller, immer mehr. Springer Fachmedien Wiesbaden, 2013. 97-112.

10 Schade V, Graf N, Bühler R, Sturzenegger M. Case-Management in der Neurologie : Entlastung durch Verlagerung: Inselbote. 2003;4:37-41. 